\title{
Simulation research on hypersonic vehicle based on fuzzy adaptive control
}

\author{
Zhouli Zhu ${ }^{\text {a }}$, Wei Chen ${ }^{b}$, Wei Zhou ${ }^{c}$, Qun Li ${ }^{d}$ \\ Collage of Information System and Management, National University of Defense Technology, \\ Changsha 410000, China \\ azhuzhou628@163.com, bwchenforever@foxmail.com, ‘nudt_zhouwei@163.com, \\ diqun@nudt.edu.cn
}

Keywords: Hypersonic vehicle, Fuzzy adaptive control, PID controller, MATLAB.

\begin{abstract}
Aiming at the control problem of hypersonic vehicle, the traditional linear control method fails to meet the expected control index, thus this paper establishes the control model of the aircraft. This paper applies the fuzzy control structure to the traditional PID controller, and through fuzzy control to adjust PID parameters in real-time to achieve adaptive control. And then by using MATLAB simulation, the influence of speed change and height change to the control of the aircraft is analyzed. The simulation results show that compared with the traditional PID control, the controller designed in this paper has higher robustness and better tracking effect.
\end{abstract}

\section{Introduction}

Hypersonic vehicle [1] is a new type of aircraft, its general flight speed is 5 times faster than the speed of sound. Hypersonic vehicles have advanced science and technology, and combine with aerospace and other aspects of cutting-edge technology, these technology is help to improve the use value of the military and civilian. Compared with the traditional technology, these cutting-edge technology is with more excellent features. Therefore, researching the hypersonic vehicle control methods has great military value and potentially huge economic value.

\section{Current Status of Research}

In the study of hypersonic vehicle control, the main method is classic PID control, this method has the advantages of simple design and clear idea, but it needs precise model of the object, and the control effect is not good. At present, the more mature method is sliding mode variable structure control, SMVSC, but this method is not accurate enough to control the target. There is also a fuzzy control method based on particle swarm optimization, and an adaptive linear quadratic control method [2], but it is difficult to ensure the stable operation of the system. In the field of aerospace, the most widely used is the optimal control theory, but the controller requires a very precise object mathematical model. Now, the more popular approach is nonlinear controller and intelligent control method, and it has made some good results.

In view of the problems above, this paper proposes a fuzzy adaptive PID control method [3], establishes the control model of the aircraft, designs the speed controller and the height controller separately, and applies the fuzzy control to the traditional PID controller to design fuzzy PID controller. Through fuzzy control, it is able to adjust PID parameters in real-time. For the design of the controller, the paper analyzes the influencing factors, uses the MATLAB to simulate, and compares with traditional single PID control at last. 


\section{Aircraft Model and Controller Design}

\subsection{The Model of Hypersonic Aircraft.}

The hypersonic vehicle model has the features of strong nonlinearity and strong coupling, so the non-linear model must be decoupled. In the general state, the longitudinal motion model of the hypersonic vehicle can be decoupled. The main parameters used are given below.

Table 1 Main parameters of hypersonic vehicle

\begin{tabular}{|c|c|c|c|c|c|}
\hline Symbol & Description & Symbol & Description & Symbol & Description \\
\hline$V$ & Velocity & $R_{e}$ & Earth's radius & $S$ & $\begin{array}{l}\text { Aerodynamic } \\
\text { reference area }\end{array}$ \\
\hline$h$ & Altitude & $\alpha$ & Angle of attack & $M$ & Mach number \\
\hline$\gamma$ & Flight-path angle & $T$ & Thrust & $\bar{q}$ & $\begin{array}{l}\text { Pneumatic } \\
\text { pressure }\end{array}$ \\
\hline$D$ & Drag & $L$ & Lift & $C_{L}$ & Lift coefficient \\
\hline$\delta_{e}$ & $\begin{array}{l}\text { Elevator deflection } \\
\text { angle }\end{array}$ & $M_{y y}$ & Pitching moment & $m$ & Quality of aircraft \\
\hline$\beta_{c}$ & $\begin{array}{l}\text { Throttle } \\
\text { setting }\end{array}$ & $I_{y y}$ & $\begin{array}{l}\text { Pitch moment of } \\
\text { inertia }\end{array}$ & $C_{D}$ & $\begin{array}{l}\text { Resistance } \\
\text { coefficient }\end{array}$ \\
\hline $\bar{c}$ & $\begin{array}{l}\text { Pneumatic chord } \\
\text { length }\end{array}$ & $C_{T}$ & Thrust coefficient & $q$ & Pitch rate \\
\hline
\end{tabular}

A nonlinear model for the longitudinal dynamics of a hypersonic aircraft on particular flight condition is presented in references $[4,5]$. The longitudinal dynamics of the air vehicle model can be described by a set of differential equations for velocity, flight-path angle, altitude, angle of attack, and pitch rate as follow:

$$
\begin{aligned}
& \dot{V}=\frac{T \cos \alpha-D}{m}-\frac{\mu \sin \gamma}{r^{2}} \\
& \dot{\gamma}=\frac{L+T \sin \alpha}{m V}-\frac{\mu-V r \cos \gamma}{V r^{2}} \\
& \dot{h}=V \sin \gamma \\
& \dot{\alpha}=q-\dot{\gamma} \\
& \dot{q}=\frac{M_{y y}}{I_{y y}}
\end{aligned}
$$

Where: $L=\bar{q} S C_{L}, D=\bar{q} S C_{D}, T=\bar{q} S C_{T}, M_{y y}=\bar{q} S \bar{C}\left[C_{M}(\alpha)+C_{M}\left(\delta_{E}\right)+C_{M}(q)\right]$ and $r=h+R_{e}$.

From the reference [6], we can get the multiple expressions of $C_{L}, C_{D}, C_{T}$ and $C_{M}$ about $M, \alpha, \delta_{e}$ and $\beta_{c}: C_{L}=0.6203 \alpha, C_{D}=0.6450 \alpha^{2}+0.0043378 \alpha+0.003772$.

$$
\begin{aligned}
& C_{T}=\left\{\begin{array}{l}
0.02576 \beta, \beta<1 \\
0.0224+0.003363 \beta, \beta>1
\end{array}\right. \\
& C_{M}(q)=(\bar{c} / 2 V) *\left(-6.796 \alpha^{2}+0.3015 \alpha-0.2289\right) \\
& C_{M}(\alpha)=0.035 \alpha^{2}+0.036617 \alpha+5.3261 \times 10^{-6} \\
& C_{M}\left(\delta_{E}\right)=c_{e}\left(\delta_{E}-\alpha\right)
\end{aligned}
$$




\subsection{Fuzzy Controller Design}

This paper designs a fuzzy adaptive PID controller [7] to improve the control accuracy and dynamic quality of the object. In the large error range, using fuzzy adaptive control can adjust PID parameters in real-time to reduce the system error quickly, and can also shorten the response time of the system. Through fuzzy rules, changing the PID parameters to control the controlled object. The simulation block diagram shown in Figure 1.

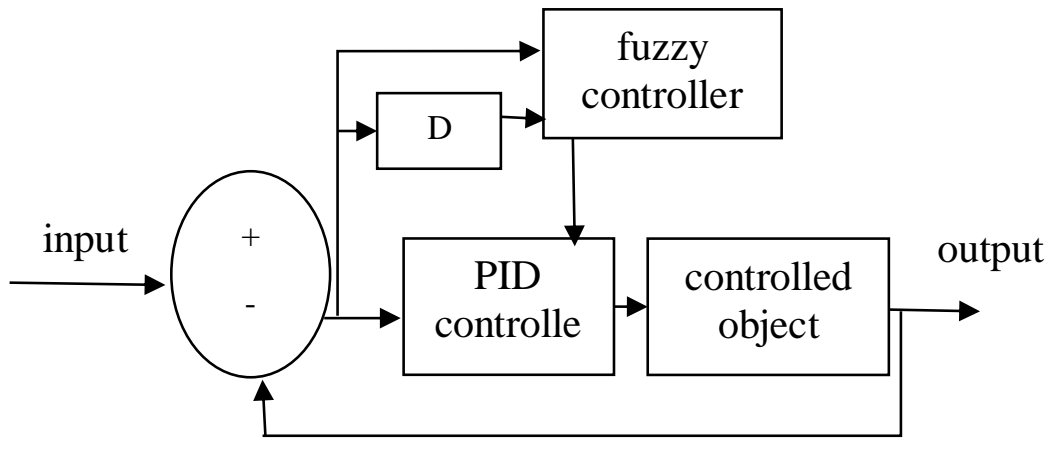

Fig. 1 Fuzzy PID simulation model

The design of the fuzzy controller structure mainly includes the following steps:

Fuzzifying the input;

Creating a database;

Designing fuzzy rule;

Fuzzy reasoning and removing the fuzzification.

The input error of the fuzzy controller is e, and error rate of change is $e_{c}$, the output is the change of the PID parameter $\Delta \mathrm{kp}, \Delta \mathrm{ki}, \Delta \mathrm{kd}$. First, fuzzifying input of the fuzzy controller. Then, putting the variable e and $e_{c}$ as the input of the fuzzy controller, the output is $\Delta \mathrm{kp}, \Delta \mathrm{ki}$ and $\Delta \mathrm{kd}$, and their language value is NB, NM, NS, Z, PS, PM, PB. The membership function of input and output variables is shown in Figure 2.

The fuzzy controller uses the fuzzy logic system of the IF-THEN rule, where the i-th rule is $\gamma^{i}$ : IF $x_{1}$ is $R_{1}^{i}$ and $x_{2}$ is $R_{2}^{i}$ and $\ldots$ and $x_{n}$ is $R_{n}^{i}$, THAN y is $u_{i},(\mathrm{i}=1 \ldots \mathrm{n})$. Where $\mathrm{n}$ is the number of fuzzy rules. Through the summary of the operational experience and the processing of the operational data, combined with the theoretical analysis, we can sum up the relationship between input e and $e_{c}$ with kp, ki and kd [8]:

When e ( $t$ ) is small, in order to make the system has a good stability, we choose a larger $\quad k p$ and ki; At the same time, in order to avoid the system appearing oscillation in the equilibrium point, the value of kd should be appropriate.

When e ( $\mathrm{t}$ ) is in the medium size, kp should be smaller to keep the overshoot smaller; In order to ensure that the system response speed will not be too slow, the value of kd should be appropriate and we can increase the value of ki.

When e $(t)$ is large, in order to speed up the system response speed, kp should be larger; but in order to reduce the system's overshoot, kd should be smaller.

The role of the proportion is to eliminate the error, the greater the proportion value is, the faster the speed is, and the smaller the error is. The integral function is to eliminate the steady-state error of the system. Differential can reduce the overshoot, and has a good control effect to the large lagged system. According to the summary above, we can get the relationship between the input variable e and $e_{c}$ with the PID parameter. Combined with practical experience and related analysis, we can get fuzzy rules table shown in Figure 3. 


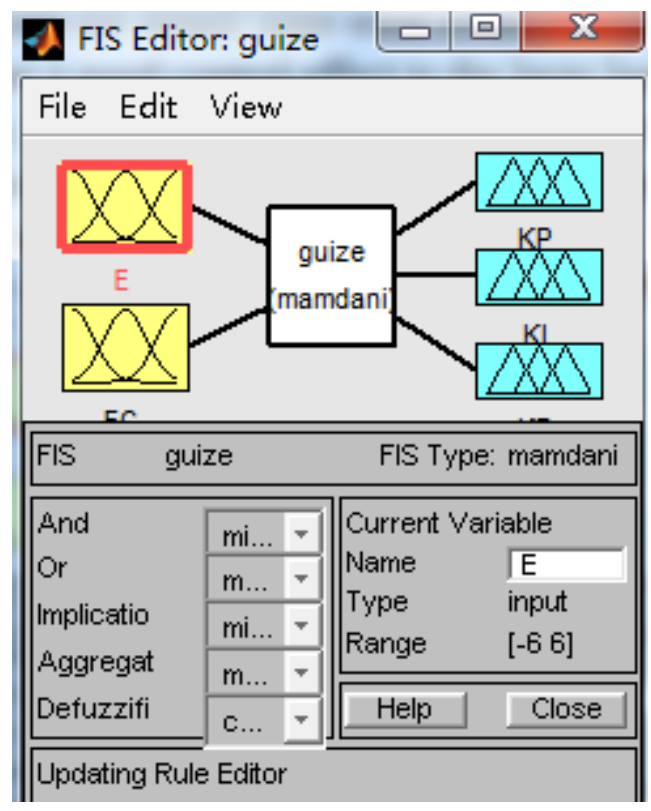

Fig. 2 Membership function of fuzzy controller's input and output variables

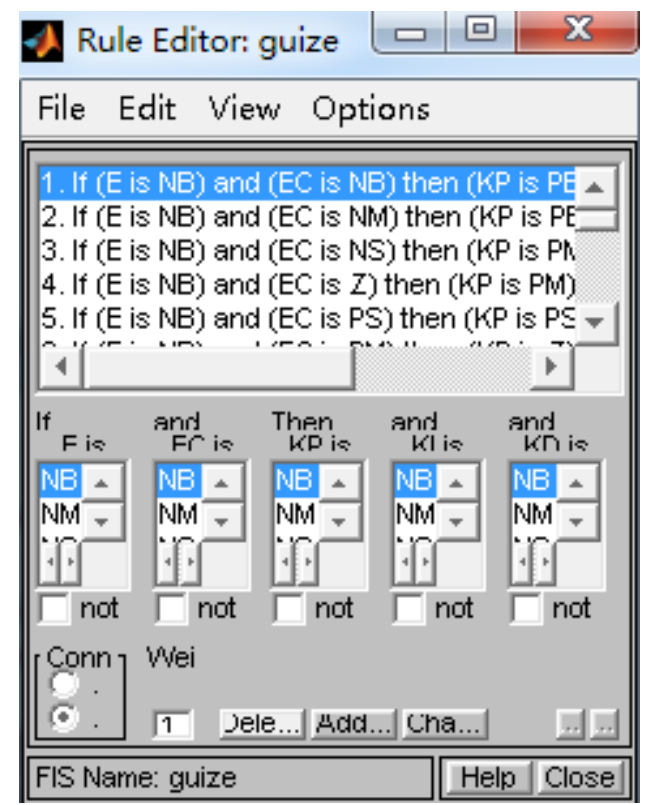

Fig. 3 Fuzzy rule table of kp, ki and kd

\section{Simulation and Results Analysis}

In this paper, the hypersonic vehicle model's initial state is $\mathrm{M}=15, V_{0}=4550 \mathrm{~m} / \mathrm{s}, h_{0}=33528 \mathrm{~m}$, $\gamma_{0}=0 \mathrm{rad}, q_{0}=0 \mathrm{rad} / \mathrm{s}, \alpha_{0}=0.0314 \mathrm{rad}, \beta_{c 0}=0.2, \delta_{e 0}=-0.00698 \mathrm{rad}$.

\subsection{Velocity tracking.}

First, we make a simulation to the fuzzy adaptive PID controller designed in this paper, and compares the results with the traditional PID controller, the results are shown in Figure 4. It can be seen from the figure that PID controller has an overshoot on the speed tracking. The adjustment time is also greater than the fuzzy adaptive controller. It is further explained that the fuzzy PID controller has good control effect and tracking ability to the model. Then we make a slope signal as input to get results of Speed tracking simulation again, and the result is shown as Figure 5. From the figure, it is clear that the fuzzy PID controller has a better control effect of tracking ability than the traditional PID. 


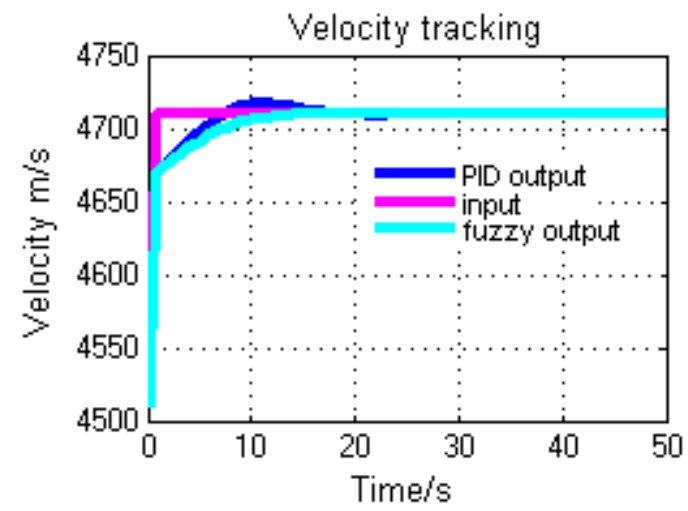

Fig. 4 The comparison between fuzzy PID control and traditional PID control under the step signal input

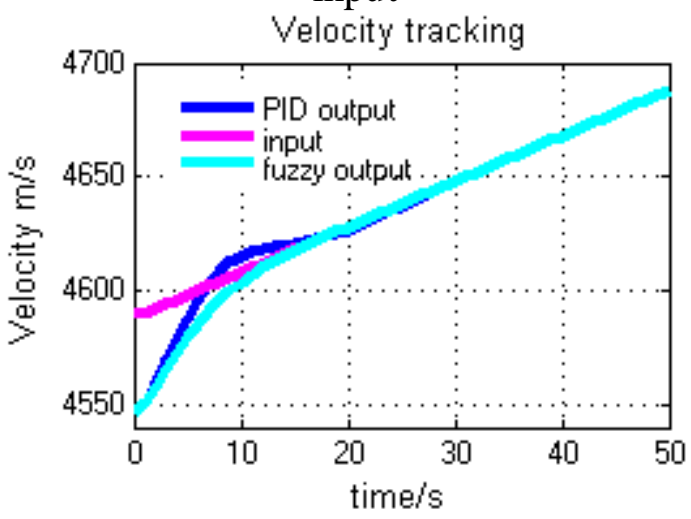

Fig. 5 The comparison between fuzzy PID control and traditional PID control under the ramp signal

\subsection{Altitude tracking.} input

As for height control, the traditional PID controller and fuzzy adaptive PID controller have a good control effect, both of them have no overshoot, but traditional PID responses slowly to the height, and it takes a long time to achieve a steady state. On the other hand, fuzzy adaptive PID has a shorter adjustment time then traditional PID controller, and the adjustment process is smooth. Thus we can see that the controller can accurately track the object in a short time. The fuzzy adaptive PID controller can adapt well to the change of the hypersonic vehicle model's parameters, and it has strong robustness and adaptability to the change of the model.

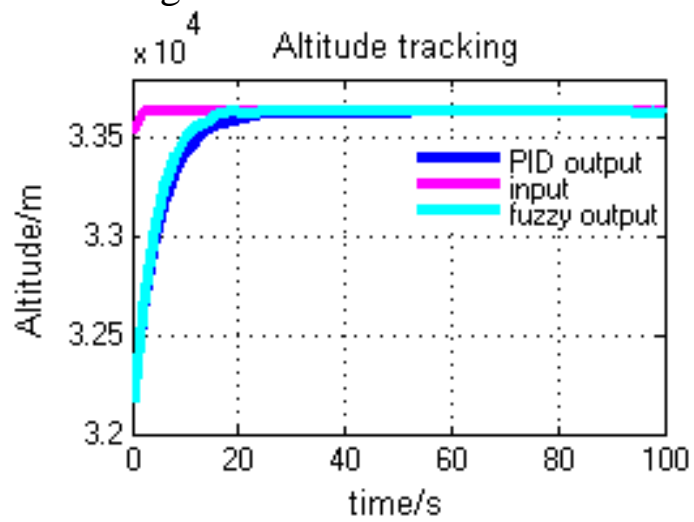

Fig. 6 The comparison between fuzzy PID control and traditional PID control under the ramp signal input 


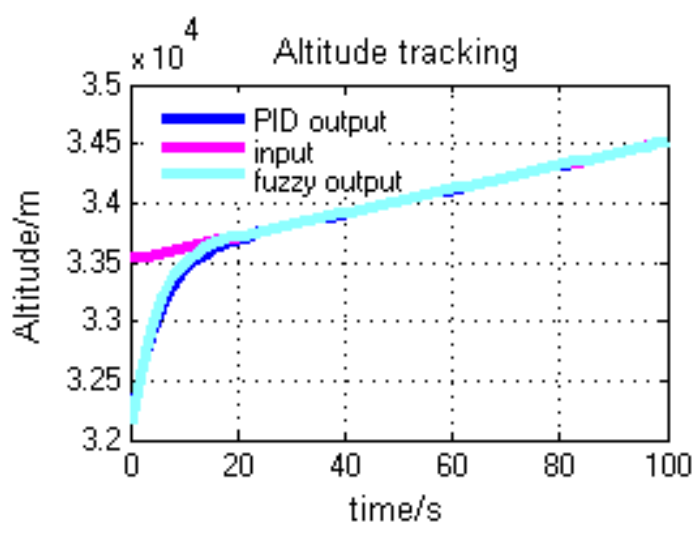

Fig. 7 The comparison between fuzzy PID control and traditional PID control under the ramp signal input

\section{Summary and Outlook}

This paper establishes the control model of the aircraft, designs the speed controller and height controller separately, and applies the fuzzy control to the traditional PID controller to design fuzzy PID controller. Through fuzzy control, it is able to adjust PID parameters in real-time. In the paper, we analyze the influencing factors, use the MATLAB to simulate, and compare with traditional PID control at last. Simulation results show that whether for the step input signal or ramp input signal, the system adjustment effect is very good. Whether for the speed control or height control, the fuzzy adaptive control effect is also better than the traditional PID control effect. In particular, as for the quick response, the fuzzy PID control's time is shorter, and the control is smooth. And the method can reduce the overshoot. In speed control, there is almost no overshoot; In height control, the final steady-state error is smaller. So the fuzzy PID controller has a better control effect than traditional PID controller, and it is more accurate to get close to the actual model of the controlled object.

Although the controller has a better control effect, the object model is simplified, so it may have a certain impact on the results obtained. The variables analyzed in this paper are not too much and it may have some deviation to the actual control. In view of the problems above, the paper proposes the following suggestions:

As the hypersonic vehicle flight conditions are complex, the dynamic structure and parameters of the aircraft are easy changed, so we should get the exact parameters of the object.

Finding a more efficient controller, such as the intelligence control, improving the accuracy of the control algorithm to achieve better control effect.

Combining with the most advanced control technology at home and abroad, studying and finding an optimal control effect to improve the control technology.

\section{References}

[1].Wei J L, Yu Y F, Yan J. Research on robust control method of hypersonic vehicle. Journal of Astronautics. Vol. 29 (2008) No. 5, p. 1526-1530.

[2].Zhou C, Zhang H H. Model Linearization and Pole-placement Design of Hypersonic Vehicle. Chinese Journal of Scientific Instrument. Vol. 29 (2008) No. 8, p. 364-367.

[3].Liu T, Li Y, Yan B B, Yu Y F. Design of Control for Hypersonic Vehicle Based on FuzzyAdaptive. Science Technology and Engineering. Vol. 13 (2013) No. 30, p. 8966-8970.

[4].Parker J T, Serrani A, Yurkovich S. Control-oriented modeling of an air-breathing hypersonic vehicle. Journal of Guidance, Control, and Dynamics. Vol. 30 (2007) No. 3, p. 856-869.

[5].Li X G, Fang Q. Winged missile flight dynamics. Xi'an: Northwestern Polytechnical University Press, 2005, p. 1-70.

[6].Li S G. The development status of hypersonic spacecraft and correlative technology research abroad. Aerospace Manufacturing Technology. Vol. 6 (2007) p. 1-5. 
[7].Meng Z J, Fu W X, Chen K, Yan J. Robust Control Design for a Hypersonic Vehicle. Journal of Projectiles. Vol. 29 (2009) No. 2, p. 12-15.

[8].Shi X M, Hao Z Q. Fuzzy Control and MATLAB Simulation. Beijing: Tsinghua University Press; Beijing Jiaotong University Press, 2008.

[9].Shi Z K, Wu F X. The robust control theory. Beijing: National Defense Industry Press, 2003, p. 187- 231. 